\title{
Research and Design of cement enterprise energy management system Report Generation
}

\author{
Dongyuan Cheng ${ }^{1, a,{ }^{*}}$, Qingjin Meng ${ }^{2, b}$ and Shaohong Jing ${ }^{2, c}$ \\ ${ }^{1}$ School of Electrical Engineering University of Jinan, Jinan 250022, China \\ ${ }^{2}$ CVIC Software Engineering Co., Ltd, Jinan 250014, China \\ a18254136693@163.com, bcse_mqj@ujn.edu.cn, ccse_jsh@ujn.edu.cn
}

Keywords:XML, jQuery EasyUI, report.

\begin{abstract}
In the cement enterprise energy management reporting system plays a very important role, with analysis and comparison function cement energy data. The system uses XML technology and jQuery EasyUI plugin implements which a software development may accelerate reporting production systems. This article describes the main structure of the system, and introduces the format templates, data templates and jQuery EasyUI data parser implementation.
\end{abstract}

\section{Introduction}

Energy managementsystem to reduce energyconsumption energy saving enterprises play an important role in the cement business, whereas the system can report energy consumption data in a clear and intuitive way to present to the operator or manager. It's very easy to find out what caused the energy consumption too much reason to production management brought great convenience, but also for production scheduling and personnel management provides reference information. But the traditionalformat ofa singlereporting system, maintaining a hugeamountofcement companiesin differentreport formatsvary,according to the traditionalapproach requiresapplication developerstodevelopindividually customizedfor eachcement companiesreporting system. Especially whenchangecan't be goodbusinessto adapt to change, then you need a programmerto rewritethe programto adapt tothe new requirementsofthe report, it will increase thedifficulty andthe progress ofdevelopmentprojects [1]. Therefore,reporting systems havean important roleforreusablecompanies.

Now many companiesuse existingreportingsoftware to developreporting system, such asoverseashaveCrystal Report, Formula One, BRIO, BO, etc. It'smore populardomesticdry-run, the number ofgiant, UFIDAChinese table, or using theircontrol.But theChinesetable stylecomplex, althoughthe softwarecan completefunctional requirements, but it is difficultto meet thecustomer's userexperience, butexpensive.

\section{Key Technologies}

\subsection{XML Technology.}

$\mathrm{XML}$ is an extensible markup language refers to,and is a markup language. It was very similar to HTML in most web applications. XML is used to transmit data, while HTML is used to format and display the data. Format and content of XML documents are separate, which the same content can be displayed in a different style [2].XML stored in plain text, thus it provided an independent software and hardware data storage methods. This allows to create different applications can share data easier [3].

\section{JQuery Easy UI Plugin.}

EasyUI is based on jQuery UI plug-in collection. It does not need you to write a lot of javascript code, and just write small HTML code to define the simple and beautiful user interface. Secondly EasyUI perfect supports HTML5.EasyUI can save a lot of time for your web development to achieve good compatibility [4].This system is mainly used EasyUI plug-in is DataGrid and TreeGrid plugins. 


\subsection{System Design and Implementation.}

System's Structure.The overallarchitectureof the systemas shown below:

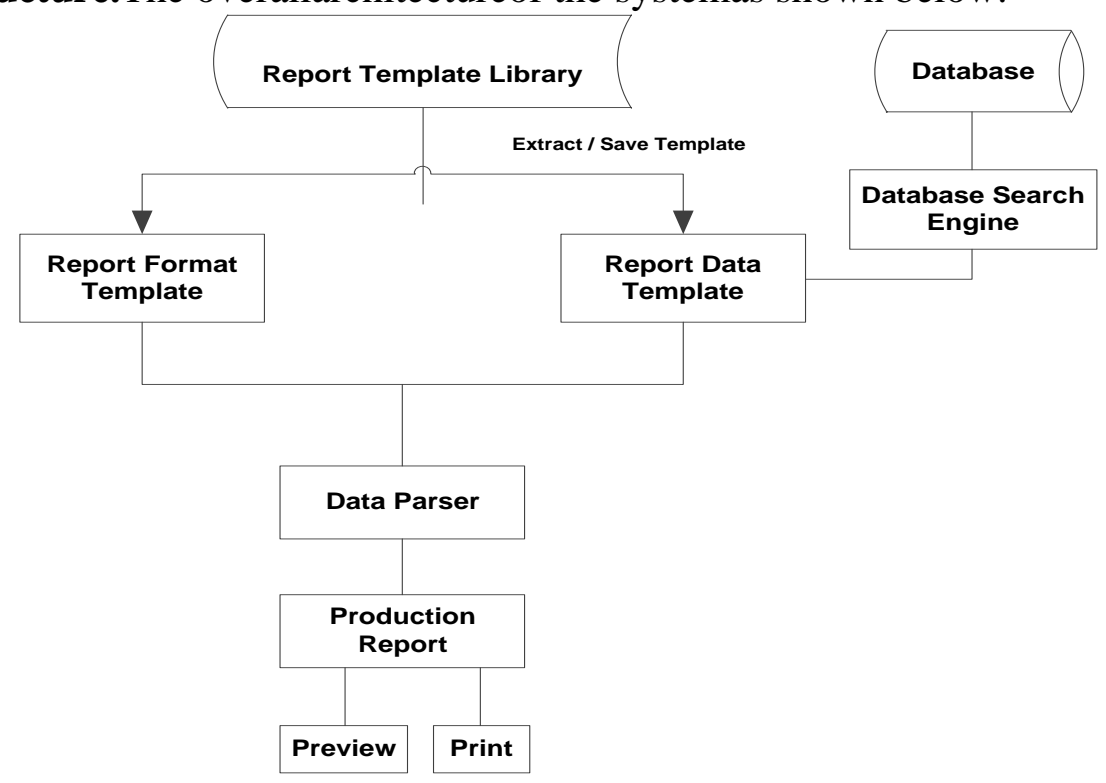

Fig.1 Overall system architecturediagram

The system design goal is to quickly complete report design work on a user-friendlyplatform. There port template library by the report templates and reports data form at template composed of both template sarede scribed by XMLlanguage. Data parserto combine these two templates to generate reports json data form at required. Thedatabase searchingengineis responsible forreading theinformation from the dataquerytemplate, and thenexecuteSQLqueriesto query data fromtakenfrom the database. The data flow shown in Figure 2:

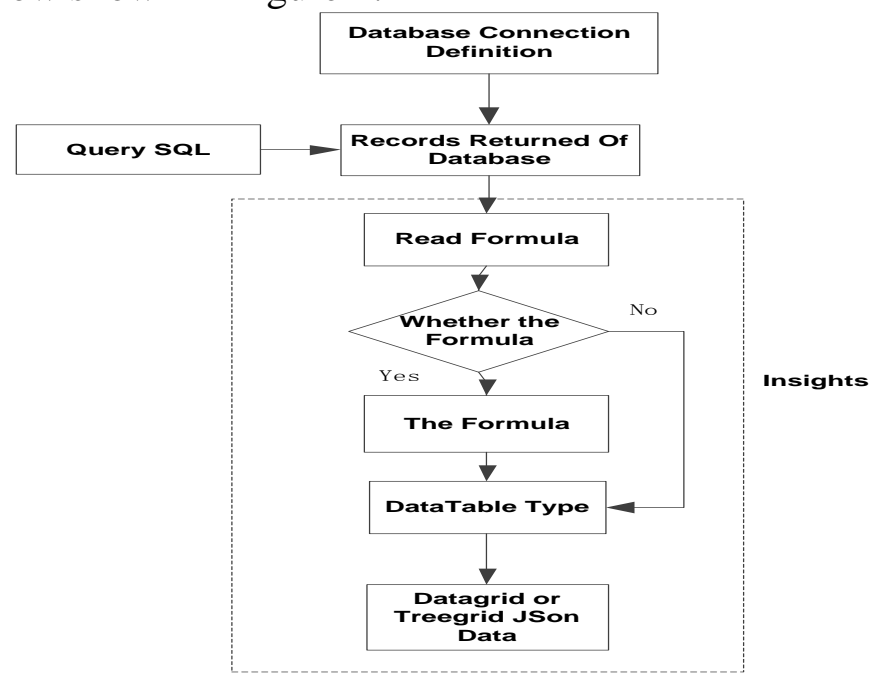

Fig.2 System data flow

Design ReportTemplate.The mainmoduleof the system include: reportformatdesign module, report datatemplate module, report dataanalysis module

Report FormatModule.Traditional reportingsystem formatdesign requirementsin the programaredesignedspecificallyfor eachstyle, such as a merger, the column widthis set, bindingthe report headerinformationinthe cellsofthe fieldshouldbe set byprogramming. When users need tochange thereporting styletablemustmodifysource code, increasing the difficulty of system maintenance.

Thesystem can be usedto generatethe requiredExcelreporting style, adjust the width ofthe cell, and the need to mergethe cellsin Excelto adjust,fill inthe fieldsof informationneed to be boundin adownwardheader.There areformulasalso addspecificformula to theappropriate cell. Then the finalformattemplate generatorwillbe loadedintoExcelformat templatebuilder,choose a goodheaderof theregionand bindthe appropriate fields, and thenconvertedto the desiredExcelXMLfile. 
Figure 3 is "Energy consumption Month Analysis Report" Format Template Builder screenshots.

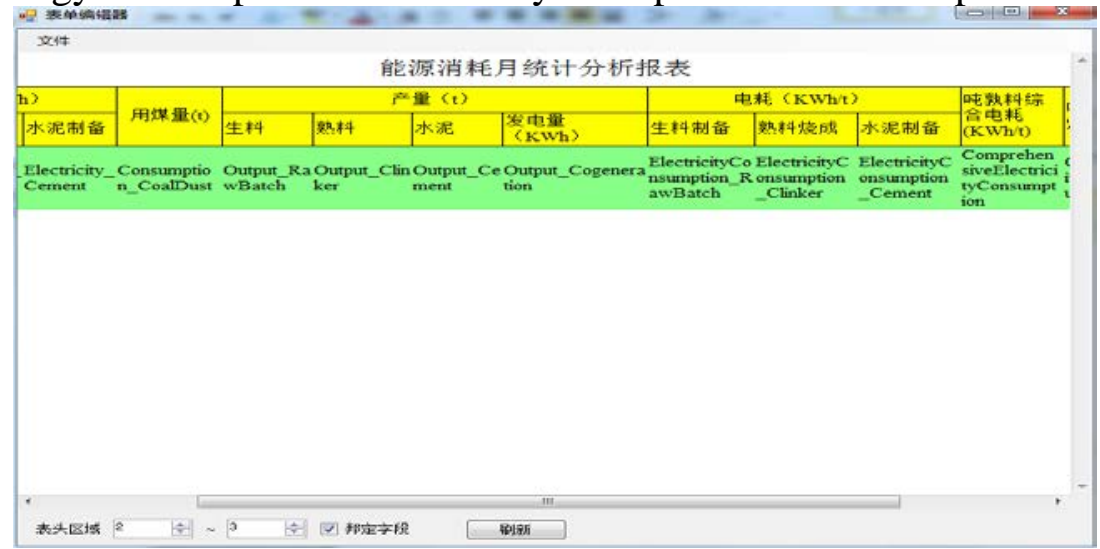

Fig.3FormatTemplate Builderscreenshot

Exported XML file formats:

$<$ mForm $>$

$<$ mRows $>$

$<$ FormColumn $>$

$<$ mBorderLeftWidth $>0</$ mBorderLeftWidth $>$

$<$ mBorderTopWidth $>0</$ mBorderTopWidth $>$

$<$ mBorderRightWidth $>0<$ mBorderRightWidth $>$

$<$ mBorderBottomWidth $>0</$ mBorderBottomWidth $>$

$<$ mWidth $>100<$ mWidth $>$

$<$ mHeight $>13.5<$ /mHeight $>$

$<$ mFontSize $>11</$ mFontSize $>$

$<$ mTextAlign $>1<$ mTextAlign $>$

$<$ mValign $>-4107</$ mValign $>$

$<$ mFontColor $>0</$ mFontColor $>$

$<$ mRowspan $>2<$ /mRowspan $>$

$<$ mColspan $>1</$ mColspan $>$

$<$ mData $>$ Coal consumption $<$ mData $>$

$<$ bindField type="field" $>$ CoalDustConsumption $<$ /bindField $>$

$<$ mDataType $>$ decimal $</$ mDataType $>$

$</$ FormColumn $>$

$<$ FormColumn $>$...

$<$ FormColumn $>$

$</$ mRows $>$

$</$ mForm $>$

$<$ mRows $>$ elementnodeis a row header, <FormColumn $>$ node is acolumn cellelement, $<$ FormColumn $>$ element and contains a description ofthe nextcellinformationsub-elements, withspecific instructionsto dothe above example: $<\boldsymbol{m}$ Width $>$ nodedigital100indicates that the column width of 100px, $<\boldsymbol{m H e i g h t}>$ digitalnode13.5indicates that thecellheight100px, $<\mathbf{m F o n t S i z e}>$ node stores the font size, $<\boldsymbol{m F o n t C o l o r}>$ node stores the font color information, $<\boldsymbol{m R o w s p a n}>$ nodem emorycells across the number of rows, the number is2 illustratesspanstwo rows, $<$ mColspan $>$ number of columnsacrossnodes in a storagecellof 1indicates thatthe cellis not listedmerger, $<\boldsymbol{m D a t a}>$ node is thecontents of the cellto be displayed,the exampleas "the amount of coal" column, the columndatatype isdecimaltype, <mDataType >node storesthedatatype of the column.

Ifthe timeis bound to aformularather than a specificfield; <bindField type = "field"> CoalDustConsumption $</$ bindField $>$ type attributenode is"field"description of the columnis bound to afield instead ofthe formula, the field is Coal Dust Consumption property typeneeds to be set "formula".

Reporting Data Template. Report data template XML file format is as follows:

$<$ Data $>$ 


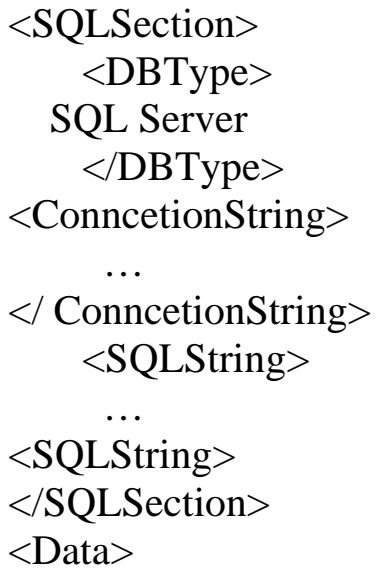

$<$ SQLSection $>$ is theSQLportion of the datatemplate, its child nodes $<$ DBType $>$ stores thetype of database, the aboveXMLfragment<DBType $>$ node datafor the"SQL Server"Description Databasetype toconnecttoSQL Servertype; <ConncetionString>information is storedinthe databaseconnection string, $<\boldsymbol{S Q L S t r i n g}>$ node storestheSQLstatement to be executed.

SQLdatabase searchenginefirst readspart of the connectioninformation about the database, based ondifferent databaseconnectiontype selection<DBType $>$ nodedatabasedescribedsuitable method, after readingthe connection string information<ConncetionString $>$ node, and establisha databaseconnection, and finallyexecuteSQLquery that returnsdatabase queryrecords.

Database searchengine applicationdesign patternsof the factory model, and the application ofreflectivetechnology that enablesdifferent types ofdatabaseoperations, meetobject-oriented designprinciples ofopening and closing.

\subsection{Implementation of Data Parser.}

Easy UID at a Form plug (datagrid) to display data in a tabular format, and provides a wealthofsupportfor the selection, sorting, groupingand editing data. Tree Form (treegrid)is used to display hierarchical datain a grid, which is based on the data table (datagrid), combined with the treeview (treeview)and editablegrid.TreeForm(treegrid)allows you to createcustomizable,expandable rowasynchronouslyand display hierarchical datainmulti-columnformat. They have their own specific JSON dataformat. By designing two dedicated C\#class that encapsulates the data conversion, Data GridJson Parserclass encapsulates the desired typefrom Data Table to Datagrid type of conversion; Tree GridJson Parser class encapsulates the data type conversion from Data Table typerequired to Treegrid.

DataGridJsonParserclassprovides the followinginterfaces: Publicstaticstring DataTableToJson (DataTable table, paramsstringcolumnsToParse);

Public static string DataTableToJson (DataTable table, intmyRowCount, params stringcolumnsToParse);

\section{DataGridJsonParserclassprovides the followinginterfaces:}

Public static string DataTableToJsonByLevelCode(Data TablemyTable, string levelCodeColumn, params string columnsToParse);

public static string DataTableToJson(DataTabletable, string groupBy, params string[] columnsToParse);

Public static string DataTableToJson (DataTable table, string idColumn, string parentIdColumn, params string columnsToParse);

\section{Applications}

In currently, this system has been well used in domesticlarge-scaleenterprises for cement. This system compared with the previous reporting system to improve the flexibility of the system and reduce the operating person neland report query reporting staff. And systemis purpose for finding outtheresult interms ofhigh energy consumption, reducing the energy consumption of cement enterprises, improving the cement business benefits. In addition, this system is based on 
$\mathrm{B} /$ Sarchitecture and convenient, eliminating the C/Sarchitecturetedious configuration and maintenance and up gradecosts.

\section{Summary}

Based on thecurrentproduction systemon the basis of the current report analyzes the proposed XML-based report generationsystemof ideas. And through XML technology and jQuery Easy UIplug-in implements the basic function son the basis of the reportbut also to meetthe requirements ofcomplex Chinesereports.

\section{Acknowledgements}

This work was financially supported byMajor projectsofShandong Province independent innovation achievements(2014CGZH0601) andChina-EU SMEs Cooperation Fund for energy conservation research project (SQ2013ZOC600003).

\section{References}

[1] Sun Huifen. The design and implementation of [J]. system based on NET environment. 2011, 07 (35). DOI:10.3969/j.issn.1009-3044.2011.35.053.

[2] Sun Huifen. The design and implementation of [J]. system based on NET environment. 2011, 07 (35). DOI:10.3969/j.issn.1009-3044.2011.35.053.

[3] Wang Xin, Liu Guangshuai, Qin Fuyang. Design and implementation of Web report generation system [J]. coal science and technology, 2006, 34 (12): DOI:10.3969/j.issn.0253-2336.2006.12.017. 47-49.

[4] Wang Xin, Liu Guangshuai, Qin Fuyang. Design and implementation of Web report generation system [J]. coal science and technology, 2006, 34 (12): DOI:10.3969/j.issn.0253-2336.2006.12.017. 47-49. 